\title{
Common dysregulation of Wnt/Frizzled receptor elements in human hepatocellular carcinoma
}

\section{A Bengochea ${ }^{1,2}$, MM de Souza ${ }^{1,2}$, L Lefrançois ${ }^{1,2}$, E Le Roux ${ }^{3}$, O Galy ${ }^{1,2}, I_{\text {Chemin }}^{1,2}, M^{K_{i m}}{ }^{4}$, JR Wands ${ }^{4}$,

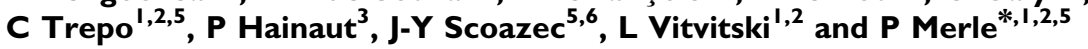

'INSERM, U87I, Molecular Physiopathology and New Therapies in Viral Hepatitis, 15 I cours Albert Thomas, Lyon, F-69424, France; ${ }^{2}$ Université Claude Bernard Lyon-I, Faculté de Médecine Laennec, IFR62, Lyon, F-69008, France; ${ }^{3}$ IARC, Molecular Carcinogenesis, Lyon, France; ${ }^{4}$ Department of Medicine, The Liver Research Center, Brown Medical School, Providence, Rhode Island, USA; ${ }^{5}$ Hepatology Unit, Hospices Civils de Lyon, Hotel-Dieu Hospital, Lyon, F-69002, France; ${ }^{6}$ Anatomopathology Laboratory, Edouard Hérriot Hospital, Lyon, France

Dysregulation of growth factors and their receptors is central to human hepatocellular carcinoma (HCC). We previously demonstrated that the Frizzled-7 membrane receptor mediating the Wnt signalling can activate the $\beta$-catenin pathway and promotes malignancy in human hepatitis B virus-related HCCs. Expression patterns of all the 10 Frizzled receptors, and their extracellular soluble autoparacrine regulators ( 19 Wnt activators and 4 sFRP inhibitors) were assessed by real-time RT-PCR in 62 human HCC of different etiologies and their matched peritumorous areas. Immunostaining was performed to localise Frizzled on cell types in liver tissues. Regulation of three known Frizzled-dependent pathways ( $\beta$-catenin, protein kinase $\mathrm{C}$, and $\mathrm{C}$-jun $\mathrm{NH}_{2}$-terminal kinase) was measured in tissues by western blot. We found that eight Frizzled-potentially activating events were pleiotropically dysregulated in $95 \%$ HCC and 68\% peritumours as compared to normal livers (upregulations of Frizzled-3/6/7 and Wnt $3 / 4 / 5 a$, or downregulation of sFRP / 15), accumulating gradually with severity of fibrosis in peritumours and loss of differentiation status in tumours. The hepatocytes supported the Wnt/Frizzled signalling since specifically overexpressing Frizzled receptors in liver tissues. Dysregulation of the eight Frizzled-potentially activating events was associated with differential activation of the three known Frizzled-dependent pathways. This study provides an extensive analysis of the Wnt/Frizzled receptor elements and reveals that the dysregulation may be one of the most common and earliest events described thus far during hepatocarcinogenesis.

British Journal of Cancer (2008) 99, |43- I50. doi:I0.1038/sj.bjc.6604422 www.bjcancer.com

Published online 24 June 2008

(c) 2008 Cancer Research UK

Keywords: hepatocellular carcinoma; WNT; Frizzled

Hepatocellular carcinoma (HCC) is one of the most frequent tumours worldwide. It develops mostly in cirrhotic livers, whose risk factors include chronic infection by hepatitis $\mathrm{B}$ and $\mathrm{C}$ viruses (HBV and $\mathrm{HCV}$ ), and nonviral liver diseases (Llovet et al, 2003; Bosch et al, 2005). Unfortunately, cellular mechanisms of hepatocarcinogenesis remain poorly understood. Initially, a variety of genetic and epigenetic alterations were detected in HCCs and to a lesser extent in preneoplastic cirrhotic livers. Later on, DNA microarray analysis led to an extensive integrative approach, leading to the identification of clusters of HCCs associated with patterns of gene expression that allow comparison between HCC phenotypes in experimental and human HCCs (Lee and Thorgeirsson, 2006). In parallel, recent progress has been achieved in understanding the essential role and function that represents dysregulation of pleiotropic growth factors - that is, the insulinlike growth factor-II (IGF-II), hepatocyte growth factor (HGF), transforming growth factor- $\alpha$ (TGF- $\alpha$ ), transforming growth factor- $\beta$ (TGF- $\beta$ ), and wingless (Wnt) - in contributing to

\footnotetext{
* Correspondence: Professor P Merle, INSERM U87I, Molecular Physiopathology and New Therapies in Viral Hepatitis, 151 cours Albert Thomas, 69424 Lyon cedex 03, France; E-mail: phmerle@lyon.inserm.fr Received II October 2007; revised 29 April 2008; accepted 29 April 2008; published online 24 June 2008
}

proliferation and antiapoptotic behaviour of HCC cells (Breuhahn et al, 2006).

Concerning the Wnt signalling cascade, the binding of one among the 19 known extracellular soluble secreted Wnt ligands (WNT) to one or more among the 10 Frizzled receptors (FZD) in cooperation or not with LRP coreceptors (low-density lipoprotein receptor (LDLR)-related protein), LRP-5 or LRP-6, can differentially lead to the activation of either the canonical $\beta$-catenin, or the noncanonical c-Jun N-terminal kinase (JNK) and protein kinase $\mathrm{C}$ (PKC) pathways, which may control the tumour phenotype. In contrast, four sFRP (secreted Frizzled-related proteins), which are extracellular soluble factors, can bind WNT and thereby downregulate their ability to activate FZD (Jones and Jomary, 2002). Different studies have clearly shown the upregulation of WNT/FZD elements in different types of cancers, highlighting their direct role in carcinogenesis as witnessed by the activation of oncogenic pathways and control of the cancerous phenotype (Tanaka et al, 1998; Kirikoshi et al, 2001; Holcombe et al, 2002; Weeraratna et al, 2002; Qiang et al, 2003; Lu et al, 2004; Zeng ZY et al, 2007). However, the expression patterns of all the WNT/FZD family members have not been extensively evaluated in human HCC so far.

We have previously shown the activation of $\beta$-catenin by overexpression of FZD7 in human HBV-related HCCs as well as in several transgenic mouse models of hepatocarcinogenesis 
(Merle et al, 2004; Merle et al, 2005). Additionally, we have shown that FZD7 can be activated in vitro by binding of the WNT3 ligand (Kim et al, 2008). In this study, the expression patterns of all 19 $W N T, 10 F Z D$, two LRP, and four $s F R P$ genes have been determined in HCCs and in their matched surrounding precancerous peritumorous areas, by comparison to normal liver (NL) tissues. This revealed that three different FZD (FZD3, 6, and 7) are commonly upregulated in HCC cells, in association with the activation of potentially FZD-dependent cascades such as the canonical $\beta$-catenin, as well as the noncanonical PKC and JNK pathways. Furthermore, these three receptors were overexpressed concomitantly with either upregulation of three Wnt agonists (WNT3, 4, and 5A) or downregulation of two Wnt antagonists (sFRP1 and 5). We propose that accumulation of these events is independent of aetiologic factors, correlates more closely with progression from cirrhosis to HCC and ultimately directly relates to the loss of differentiation of such tumours. In parallel, we have shown that the WNT/FZD/sFRP expression patterns did not correlate to the TP53 and $\beta$-catenin gene mutation status in the HCC samples, these genes being the most frequently altered tumour suppressor and proto-oncogene in HCC (Laurent-Puig and Zucman-Rossi, 2006).

\section{MATERIALS AND METHODS}

\section{Human liver tissues}

Sixty two frozen HCCs surgically resected from different individuals were obtained from Thailand (International Agency for Research on Cancer) $(n=10)$ and France (National Resource Biological Center) $(n=52)$, a written consent being obtained before surgery. Tissue samples were obtained and characterized as tumorous (T) or matched peritumorous liver parenchymas (pT). NL came from parenchyma surrounding surgically resected focal nodular hyperplasia from different individuals $(n=9)$. HCCs were due to HBV $(n=18)$ or HCV $(n=20)$ infection as defined by positivity for HBs antigen or anti-HCV antibodies in serum, respectively, whereas others were presumed as nonviral-related (NBNC) tumours $(n=24)$.

\section{Histological analysis}

Histological analysis was performed on $\mathrm{T}$ to confirm the diagnosis of HCC and to classify them as to their differentiation status. Furthermore, histology served on $\mathrm{pT}$ as a method of assessing fibrosis stage using the Metavir criteria (The French METAVIR Cooperative Study Group, 1994), and to further ensure the absence of microscopic tumour invasion.

\section{Cell lines}

The human HCC cell lines Huh7, Focus, PLC/PRF/5, Hep3B, as well as the hepatoblastoma HepG2 cell line, were grown in DMEM F-12, $1 \mathrm{X}$-MEM nonessential amino-acid solution, $200 \mathrm{~mm}$ L-glutamine, $1 \times$-sodium-pyruvate, $1 \% \quad(\mathrm{vol} / \mathrm{vol}) \quad$ penicillin/streptomycin (Invitrogen), and supplemented with $10 \%$ (vol/vol) fetal calf serum (Sigma). Human primary hepatocytes were cultured in HCM Bullet-kit hepatocytes culture medium (Cambrex).

\section{Semi-quantitative real-time RT - PCR}

We assessed the expression of the 19 WNT, 4 sFRP, 10 FZD and 2 $L R P$ genes in cell lines, and liver tissues (62 T and $62 \mathrm{pT})$ as determined by an arbitrary value (AV) Additionally, a cohort of nine NL served as controls which gave cutoff values equal to mean \pm 2 s.d. $(\alpha=0.05)$ for upregulation or downregulation of the different genes in $\mathrm{pT}$ and T. Total RNA extractions as well as cDNA synthesis were prepared as described previously (Merle et al, 2004). PCR reactions were performed by 35 cycles $\left(95^{\circ} \mathrm{C} 15 \mathrm{~s}, 60^{\circ} \mathrm{C}\right.$
$1 \mathrm{~min}$ ) using the $\mathrm{MyIQ}^{\mathrm{TM}}$ Real Time PCR Detection System (Bio-Rad) with a mix composed of $1 \mathrm{X}$-Quantitech Sybr Green PCR Kit (Qiagen), primers at either $500 \mathrm{~nm}$ (Wnt, $s F R P, F Z D$, and $L R P$ ) or $800 \mathrm{~nm}$ (18S RNA), and $12.5 \mathrm{ng} \mathrm{cDNA}$ (equivalent total RNA) from unknown samples. Each PCR run included the unknown cDNAs and a nontemplate control (RNA sample treated with DNase without RT step) to check for the absence of genomic DNA contamination. Regarding the specificity of reactions, melt curves were analysed for each sample and the corresponding PCR products of the positive controls were cloned into the $\mathrm{pCR}^{\mathbb{R}}$ 2.1 Vector (Invitrogen, Life Technology) and sequenced. Primers for $S F R P$ genes were selected using the previously described strategy (Merle et al, 2004). Primers for FZD7 and 18SrRNA were previously published (Merle et al, 2004), as well as those for Wnt, FZD (except FZD7), and LRP (Lu et al, 2004) with slight modifications to obtain identical annealing temperature and PCR amplification efficiency as for FZD7, 18S rRNA, and $s F R P$ primers (Supplementary online data no. 1). The amount of specific mRNA was quantified in unknown samples by using the comparative $\mathrm{Ct}$ method: the $\Delta \mathrm{Ct}$ values from each tissue were obtained by subtracting the values for $18 \mathrm{~S} \mathrm{Ct}$ from the $\mathrm{Ct}$ of each tested gene. One difference in $\triangle \mathrm{Ct}$ represents a two-fold difference in the level of mRNA as described in (Lu et al, 2004), this method is giving an $\mathrm{AV}$ for the expression of each gene per tissue.

\section{Immunostaining}

Immunohistochemistry was used to localise FZD3/6/7 in T and pT tissues fixed in $10 \%$ neutral-buffered formalin and embedded in paraffin. After antigen unmasking by heating slices in a microwave oven in citrate buffer at $\mathrm{pH} 6.0$ for $10 \mathrm{~min}$, the $5 \mu \mathrm{m}$ sections were incubated overnight at $4^{\circ} \mathrm{C}$ with anti-FZD3 (1/150, LifeSpan, Biosciences, LS-A4454), anti-FZD6 (1/70, LifeSpan, Biosciences, LS-A4481) or anti-FZD7 (1/200, Sigma, F3679) rabbit polyclonal antibodies. Endogenous peroxidase activity was blocked with the Peroxidase-Blocking reagent (DakoCytomation) for $10 \mathrm{~min}$ and nonspecific antibody binding was blocked with $10 \%$ skimmed milk for $30 \mathrm{~min}$ at $20^{\circ} \mathrm{C}$. The Antibody-Diluent reagent (DakoCytomation) served as a negative control under equivalent conditions in place of the primary antibody. A goat antirabbit IgG conjugated to peroxidase-labelled polymers diluted in Tris-HCl buffer (EnVision + Dual Link System Peroxidase - DakoCytomation) was added according to the manufacturer's instructions. For amplification of the reaction, a soluble antigen-antibody of horseradish peroxidase antiperoxidase developed in the rabbit (Sigma) was used at $1 / 200$ dilution for $30 \mathrm{~min}$ at $20^{\circ} \mathrm{C}$. Colorimetric reaction was developed with 3,3'-diaminobenzidine (Liquid $\mathrm{DAB}+$ Substrate Chromogen System- DakoCytomation) for 2 min. Slides were counterstained with Harris hematoxylin, dehydrated, and coverslipped with EUKITT (O Kindler GmbH \& CO, Freiburg).

\section{Protein extraction and western blotting}

We analysed by western-blotting the activaty status of the three FZD-dependent intracellular pathways - that is, $\beta$-catenin, PKC, JNK - in T showing overexpressing of FZD3, 6, and/or 7 by comparison with their matched $\mathrm{pT}$. Samples were treated with $200 \mu$ l of extraction buffer ( $30 \mathrm{~mm}$ Tris-pH 7.5, $150 \mathrm{~mm} \mathrm{NaCl}, 1 \%$ NP-40, $0.5 \% \mathrm{Na}$ deoxycholate, $0.1 \%$ SDS, $10 \%$ glycerol and $2 \mathrm{~mm}$ EDTA) containing protease inhibitors and phosphatase inhibitors (Roche Diagnostics). Protein concentration was determined with the BCA Protein Assay Kit (Pierce) using BSA as standard. Subcellular fractionations were performed as described previously (Maloney et al, 1998), and protein concentration was measured by the method of Bradford with the Bio-Rad Protein Assay Kit (Bio-Rad Laboratories, Hercules, USA) using BSA as standard. Aliquot of proteins were resolved on SDS-PAGE and transferred 
onto PVDF membranes (Amersham) by electroblotting. The membranes were blocked with $5 \%$ nonfat dry milk in Trisbuffered saline containing $0.1 \%$ Tween 20 , and then probed with an antibody targeting total $\beta$-catenin (Transduction Laboratory) or phospho-Thr41/Ser45 $\beta$-catenin (Cell Signalling), pan-PKC (Santa Cruz Technology) or pan-phospho-PKC (Cell Signalling), JNK (R\&D Systems) or phospho-JNK (R\&D Systems). The corresponding horseradish peroxidase antibodies were incubated and revealed with the chemiluminescence imaging ECL (Sigma). All of the blots were standardized for equal protein loading by Red Ponceau staining and western blotting for $\beta$-actin (Sigma).

\section{PCR/DHPLC Sequencing of CTNNB1 and TP53 Genes}

For denaturing high performance liquid chromatography (DHPLC) analysis, crude amplification products were denaturated by heating at $95^{\circ} \mathrm{C}$ then cooled to $25^{\circ} \mathrm{C}$ over $1 \mathrm{~h}$. DHPLC analysis were performed by injecting $5-8 \mu \mathrm{l}$ of PCR products into a preheated reverse-phase column (DNASep Column, Transgenomic) equilibrated with an ion pairing agent TEAA $0.1 \mathrm{M}$ (Triethylammonium acetate). DNA was removed from the column by a linear gradient of eluting buffer containing $25 \%$ acetonitrile at a constant flow rate of $0.9 \mathrm{ml} \mathrm{min}{ }^{-1}$ and with $2 \%$ per min gradient increase. Transgenomic UV-detector identified the eluted DNA at $260 \mathrm{~nm}$. All amplimers with different profile shapes or retention times were sequenced to confirm the putative sequence variations. Concerning PCR sequencing, primer pairs were designed for amplification of exons- 4 to -9 for TP53, and exon-3 for $\beta$-catenin genes (Supplementary online data no. 2). Each PCR mix contained genomic DNA, $1.5 \mathrm{~mm} \mathrm{MgCl}_{2}, 0.2 \mathrm{~mm}$ each dNTP, $0.4 \mu \mathrm{M}$ each primer, 1X-PCR Buffer (Invitrogen) and 2U Platinum ${ }^{\circledR}$ Taq DNA Polymerase (Invitrogen) for a final reaction volume of $50 \mu \mathrm{l}$. The PCR amplification for TP53 exons was performed using the following conditions: initial denaturation at $94^{\circ} \mathrm{C} 2 \mathrm{~min}, 20$ cycles $\left(94^{\circ} \mathrm{C} 45 \mathrm{~s}, 63^{\circ} \mathrm{C} 30 \mathrm{~s}\right.$, and gradual decrease of $0.5^{\circ} \mathrm{C}$ per three cycles, $\left.72^{\circ} \mathrm{C} 45 \mathrm{~s}\right)$ followed by 30 cycles $\left(94^{\circ} \mathrm{C} 45 \mathrm{~s}, 60^{\circ} \mathrm{C} 30 \mathrm{~s}\right.$, and $72^{\circ} \mathrm{C} 45 \mathrm{~s}$ ) and ending with an extension at $72^{\circ} \mathrm{C} 10 \mathrm{~min}$. The cycling profile for $\beta$-catenin exon-3 amplification was the following: initial denaturation at $95^{\circ} \mathrm{C} 2 \mathrm{~min}, 20$ cycles $\left(95^{\circ} \mathrm{C} 30 \mathrm{~s}\right.$, $56^{\circ} \mathrm{C} 30 \mathrm{~s}$, and gradual decrease of $0.4^{\circ} \mathrm{C}$ per two cycles, $72^{\circ} \mathrm{C} 30 \mathrm{~s}$ ) followed by 30 cycles $\left(95^{\circ} \mathrm{C} 30 \mathrm{~s}, 52^{\circ} \mathrm{C} 30 \mathrm{~s}\right.$, and $\left.72^{\circ} \mathrm{C} 30 \mathrm{~s}\right)$ and ending with extension at $72^{\circ} \mathrm{C} 7 \mathrm{~min}$.

\section{Statistical analysis}

The Mann-Whitney $U$-test was used with StatView software Version 5.0 (SAS Institute Inc.), and $\chi^{2}$ test when necessary. Tests were considered significant when their $P$ values were $<0.05$.

\section{RESULTS}

Expression patterns of $F Z D, L R P, W N T$, and $s F R P$ genes at the mRNA level in liver tissues by quantitative real-time RT - PCR

Three different $F Z D$ genes were found frequently upregulated in $\mathrm{T}$ and pT by comparison to NL (>cut-off; Figure 1): FZD3 (41\% T, $23 \%$ pT), FZD6 (31\% T, $8 \%$ pT), and FZD7 (33\% T, 10\% pT). By contrast, almost none of the samples showed any significant upregulation or downregulation of $L R P$ genes in $\mathrm{pT}$ or $\mathrm{T}$ tissues by comparison to NL ( $<$ or $>$ cut-off). Concerning the soluble extracellular regulators of the Frizzled membrane receptors, WNT3, WNT4, and WNT5A, potent activators, were strikingly found upregulated by comparison to NL ( $>$ cutoff value): WNT3 (39\% T, 25\% pT), WNT4 (20\% T, 16\% pT), and WNT5A (25\% T, $7 \% \mathrm{pT}$ ). Among the potential inhibitors of the Frizzled receptors, two $s F R P$ genes were found downregulated: $s F R P 1 \quad(53 \% \mathrm{~T}, 21 \%$ $\mathrm{pT})$, and $\operatorname{sFR} 5(28 \% \mathrm{~T}, 12 \% \mathrm{pT})$.
Taken together, these results demonstrated that when pooling the eight following events - that is, upregulation of potential activators (FZD3, FZD6, FZD7, WNT3, WNT4, and WNT5A) or repression of inhibitors ( $s F R P 1$ and $s F R P 5$ ) of the WNT/FZD signalling - one of them at least occurred in $68 \% \mathrm{pT}$ and $95 \% \mathrm{~T}$. Strikingly, each of these events accumulated with progression from noncirrhotic to cirrhotic tissues of peritumour areas $(0.6 \pm 0.6$ events per noncirrhotic pT vs $1.4 \pm 0.9$ events per cirrhotic pT; $P<0.01)$. They additionally carried on their accumulation within tumours $(1.4 \pm 0.9$ events per cirrhotic pT $v s 2.1 \pm 0.9$ per welldifferentiated HCC, $P<0.05$ ), and subsequently with the loss of differentiation status of tumours $(2.1 \pm 0.9$ per well-differentiated HCC $v s 3.0 \pm 1.3$ events per moderately to poorly differentiated tumour; $P<0.05$ ) (Figure 2 ).

It is of note that, we carefully evaluated by serial tissue sections that none of the $\mathrm{pT}$ with elevated WNT/FZD or decreased $s F R P$ expression levels had microscopic HCC tumour foci. Concerning relationship to aetiologic factors of HCC, with the notable exception of FZD7 which showed higher rate of upregulation in HBV vs non-HBV-related HCC (59 vs $23 \%$, CHI-2 test, $P=0.035$ ), $W N T 3 / 4 / 5 A, F Z D 3 / 6$, and $s F R P 1 / 5$ were statistically equally dysregulated between HBV, HCV, and NBNC-related HCCs.

\section{Correlation of $W N T / F Z D / s F R P$ expression to mutation status of $\beta$-Catenin and TP53 genes in HCCs}

The $\beta$-catenin and TP53 mutation status was determined in HCC and compared to WNT/FZD/sFRP expression patterns (Supplementary online data no. 3 ). The $\beta$-catenin gene was found mutated mainly in HCV-related HCC (HBV 17\%, HCV $40 \%$, and NBNC $21 \%$ ), whereas TP53 mutations did not correlate with aetiologic factors (HBV 33\%, HCV 30\%, and NBNC 13\%). There was no correlation between these mutations and a specific WNT/FZD/sFRP expression pattern in HCC.

\section{Cell specificity of Frizzled receptors and activity of Frizzled-dependent intracellular pathways}

Immunostaining allowed at identifying the specific cells, which overexpressed FZD3, FZD6, and FZD7 proteins within T and $\mathrm{pT}$ tissues. As shown in Figure 3, these three receptors were highly expressed by HCC cells in $\mathrm{T}$, and at a lesser extent by nontransformed hepatocytes in $\mathrm{pT}$, whereas they were barely or not expressed by both nonhepatocytic cells and normal hepatocytes in NL. As a confirmatory result, quantitative real-time RT PCR data showed the capability of cancerous HCC cell lines to express high steady state levels of FZD3, FZD6, and/or FZD7 mRNAs, whereas normal primary hepatocytes did not (Table 1).

Next, we assessed the activation of the downstream pathways potentially controlled by the WNT/FZD signalling - that is, $\beta$-catenin, PKC and JNK - in liver tissues. To this aim, we examined a cohort of 15 paired samples $(\mathrm{T}+\mathrm{pT})$ where the previously identified WNT3/4/5A, FZD3/6/7, sFRP1/5 events gradually accumulated from $\mathrm{pT}$ towards the matched $\mathrm{T}$, each $\mathrm{pT}$ serving as a matched negative control for the corresponding $\mathrm{T}$. As expected, most of the tested $\mathrm{T}(13 / 15,87 \%)$ showed higher activity of either $\beta$-catenin, PKC or JNK pathways than their matched $\mathrm{pT}$ (Table 2), as shown respectively by unphosphorylation of the cytosolic form of $\beta$-catenin at threonine-41/serine-45 residues, phosphorylation of PKC at serine-660 residue, and phosphorylation of JNK at threonine-183/tyrosine-185 residues (Figure 4). Before assessing the potential impact of the WNT/FZD signalling on the activity of $\beta$-catenin, we carefully checked that all tested $\mathrm{T}$ samples did not contain specific mutations within the serine/ threonine residues of the $\beta$-catenin gene. In contrast, $3 / 4(75 \%) \mathrm{T}$ devoided of accumulation of WNT/FZD events did not show increased activity of one of the three pathways in $\mathrm{T} v s \mathrm{pT}$ (Table 2). 
A

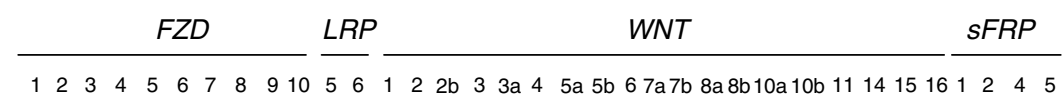

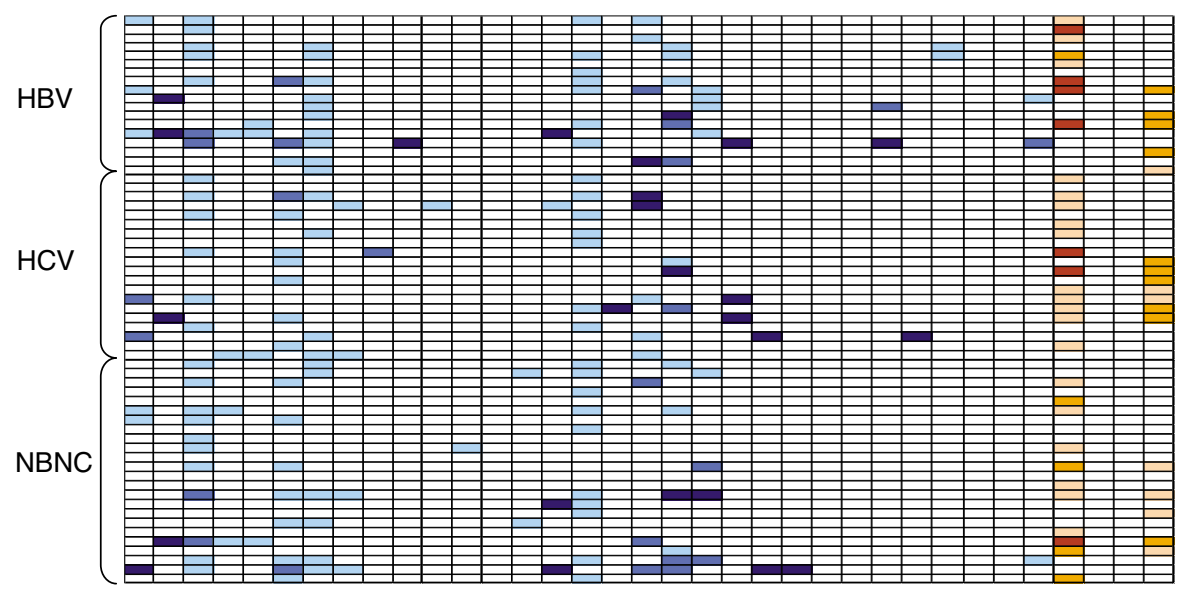

B

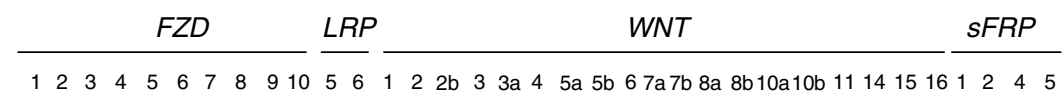

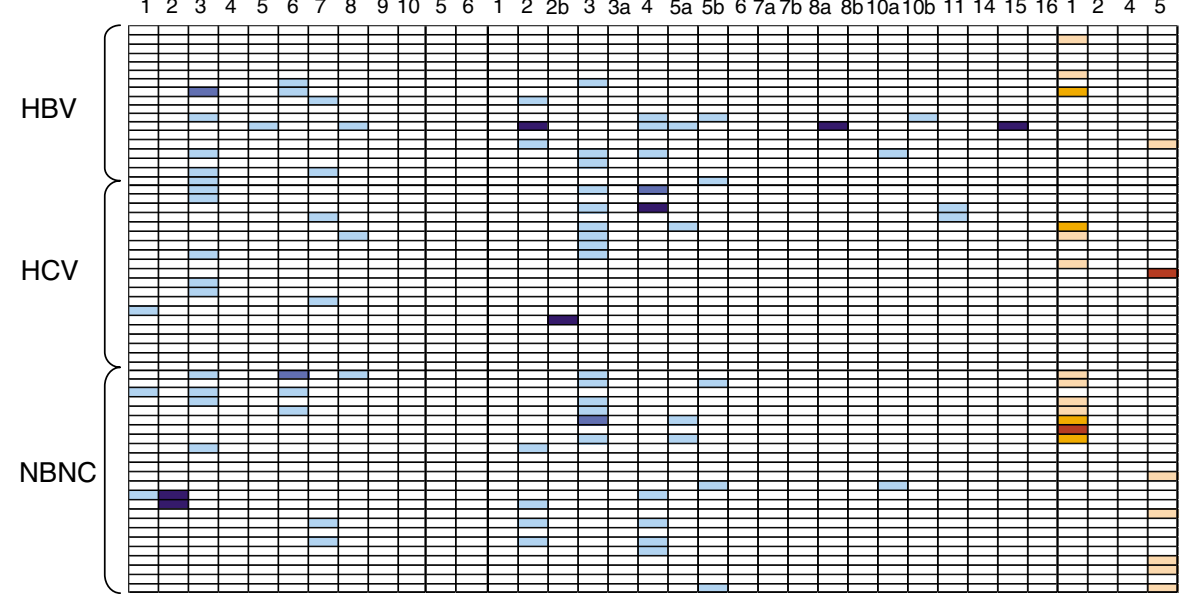

Figure I Expression patterns of FZD/LRP/WNT/SFRP genes in HCC tissues in T $(\mathbf{A})$ and $\mathrm{pT}(\mathbf{B})$, by comparison to cutoff values obtained from NL. Each line represents a different HCC tissue depending on the aetiologic factor (lines I- I8 for HBV, lines 19-38 for HCV, and lines 39-42 for NBNC). Upregulation of genes are indicated in coloured boxes as light blue (one- to five-fold), medium dark blue (five- to I0-fold), or dark blue ( $>$ I0-fold). Downregulation of genes are indicated in pink (five-fold), orange (five- to I0-fold), or brown ( $<10$-fold).

\section{DISCUSSION}

We have demonstrated for the first time that dysregulation of the WNT/FZD receptor elements is an overall common feature in human hepatocarcinogenesis. This study has provided a comprehensive transcriptomic analysis of the FZD/LRP receptor complexes, and some of their potential autoparacrine regulators - that is, WNT and sFRP elements in human HCC. We have identified eight potential FZD activating events, each occurring in a significant proportion of HCCs $(\geqslant 20 \%)$ - that is, upregulation of FZD3/6/7 and WNT3/4/5A, and repression of $s F R P 1 / s F R P 5-$ which accumulate with severity of the liver disease and tumour stage. Furthermore, we have shown that the FZD3/6/7-mediated signalling is supported by HCC cells within tumour liver tissues. Finally, we have observed that at least one over the three known different intracellular pathways potentially controlled by the WNT/FZD signalling during embryogenesis or carcinogenesis that is, canonical $\beta$-catenin, or noncanonical PKC and JNK - appeared activated in a majority of the tested HCC tissues containing the accumulation of WNT/FZD element dysregulations.

Research on dysregulation of growth factors and their receptors aims at establishing the role and function of the corresponding signalling cascades in human hepatocarcinogenesis towards the identification of new therapeutic targets. In this view, we previously demonstrated the activation of the WNT/FZD signalling through FZD7 overexpression in transgenic mouse models of HCC as well as in human HBV-related HCC tissues from Taiwan and South Africa (Merle et al, 2004, 2005). However, it remained questionable whether FZD7, among the ten known human FZD receptors, is the unique $F Z D$ involved in human hepatocarcinogenesis, and what happened in non-HBV-related HCCs. In this study, we have confirmed in tissues from Thailand and Western Europe that FZD7 is overexpressed in a majority of HBV-related HCCs and, although at a lesser extent, in a significant proportion of HCV and nonvirus-related HCCs. At present, no information is available as to the possible interactions between $\mathrm{HBV}$ and the regulatory elements of $F Z D 7$, thus emphasising the need for additional investigation. Furthermore, we have shown that not only FZD7 but also FZD3 and FZD6 are commonly overexpressed in HCCs. Noticeably, FZD3, and FZD7 were found more closely related to the poorly differentiation status of tumours, indicating their possible involvement in the latter steps of hepatocarcinogenesis, and their possible role in the metastasis processes and epithelial-mesenchymal transition as recently reported for FZD7 in 


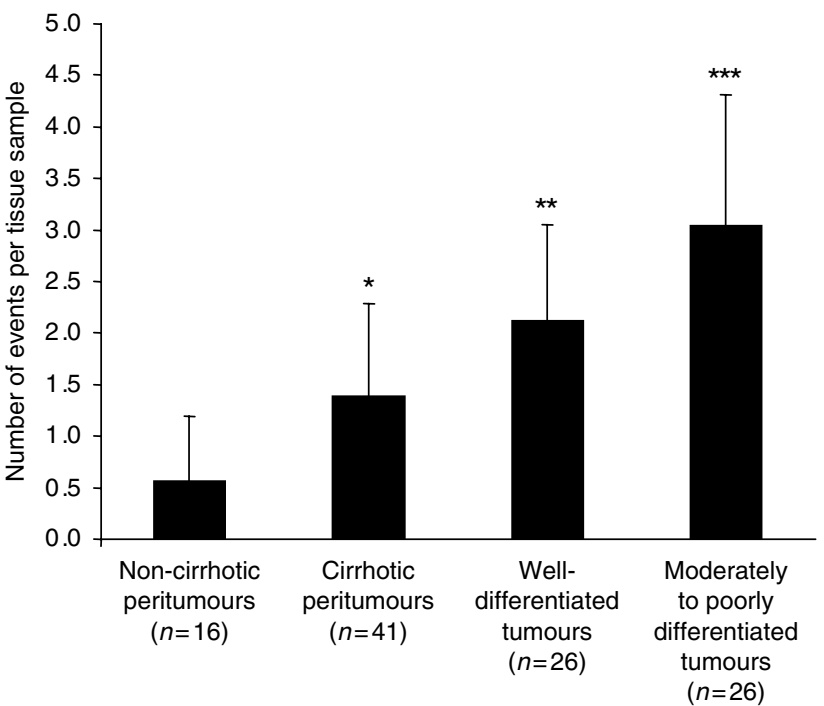

Figure 2 Evidence of accumulating WNT/FZD events with occurrence of cirrhosis in peritumorous tissues, with development of tumours and subsequently with severity of tumours in terms of dedifferentiation status. Bars ( \pm s.d.) represent the average number of events - that is, upregulation of FZD3, FZD6, FZD7, WNT3, WNT4, and WNT5A or downregulation of sFRPI, sFRP5 - per tissue sample. Comparisons were statistically assessed by the Mann-Whitney $U$-test : $* P<0.0$ I between noncirrhotic and cirrhotic peritumours; ** $P<0.05$ between cirrhotic peritumours and welldifferentiated tumours; ${ }^{*} * * P<0.05$ between well-differentiated and moderately/poorly differentiated tumours.

colon tumours (Vincan et al, 2005). The potent soluble activators of $F Z D 3 / 6 / 7$ are very likely those ligands to be found overexpressed in HCCs (WNT3/4/5A).

These data correlate with FZD3/6 and WNT3 found overexpressed in other malignancies as leukaemia, FZD7 in solid tumours like nasopharynx, oesophagus, and stomach carcinomas, WNT5A in leukaemia, melanoma, and nasopharynx carcinoma (Tanaka et al, 1998; Kirikoshi et al, 2001; Holcombe et al, 2002; Weeraratna et al, 2002; Qiang et al, 2003; Lu et al, 2004; Zeng ZY et al, 2007), whereas WNT4 has been previously found overexpressed in pancreatic cancers (Pasca di Magliano et al, 2007). Concerning the soluble activators of $F Z D$, previous studies have shown the common downregulation of $S F R P 1$ due to promoter hypermethylation in HCC as well as in colon cancer (Suzuki et al, 2004; Shih et al, 2006, 2007). In this study, we have found that although sFRP1 was commonly downregulated in $\mathrm{pT}$ and $\mathrm{T}$, another inhibitor such as SFRP5 shared the same expression pattern of repression. Finally, as the $W N T / F Z D / s F R P$ signalling elements are dysregulated in most HCCs, we have been unable to find a specific association between their expression pattern and the occurrence of either TP53 or $\beta$-catenin gene mutations, which paradoxically had been shown in previous studies as dichotomizing HCC tumours in different clusters (Laurent-Puig et al, 2001).

Of interest was the progressive accumulation of the FZD activating events (WNT3/4/5A and FZD3/6/7 upregulations and $s F R P 1 / 5$ repression) with severity of the liver disease and the tumour stage. These results suggest that the FZD activating event accumulation may contribute to enhance the activity of the downstream pathways that may control the cancerous phenotype. Thus, we tried to assess the differential activity of the various potentially FZD-dependent intracellular pathways ( $\beta$-catenin, PKC, and JNK) between HCC tumours (T) and their matched nontumorous tissues (pT). The canonical WNT signals are transduced through FZD family receptors and LRP5/LRP6 coreceptor to the $\beta$-catenin signalling cascade. In the absence of canonical WNT signalling, $\beta$-catenin combined to APC and AXIN
A

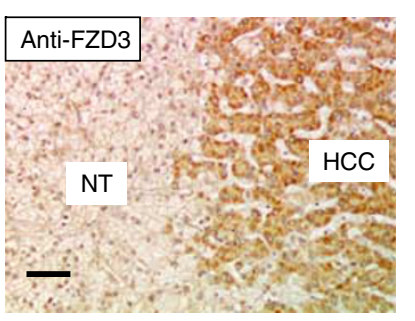

C

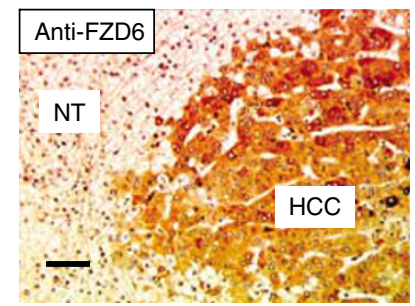

E

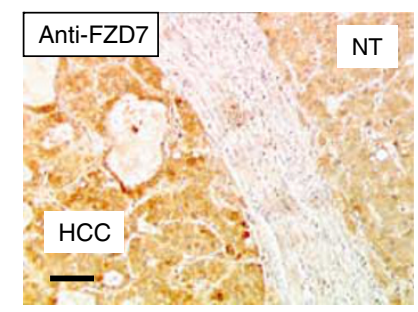

B

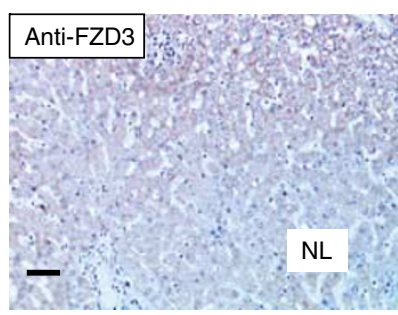

D

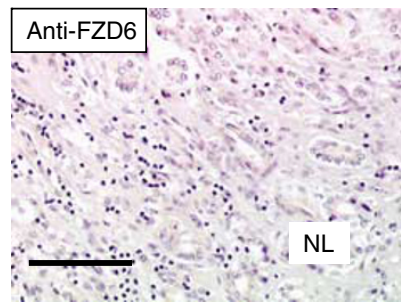

$\mathbf{F}$

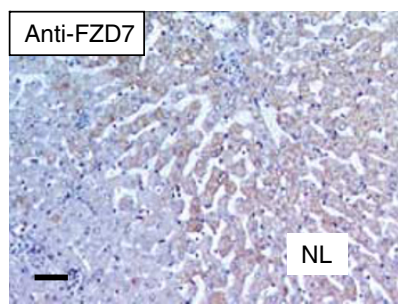

Figure 3 Representative examples of immunostaining for Frizzled receptors (FZD) in liver sections involving both $T$ and $P T$ : FZD3 (A), FZD6 (C) and FZD7 (E). Staining of NL is presented for comparison for $(\mathbf{B}, \mathbf{D}$, and $\mathbf{F})$. FZD3 was detected mainly in HCC cells as compared to adjacent nontumorous hepatocytes $(\mathbf{A})$. In the normal liver, only a faint labelling was observed in the perivenous area of the lobule (B). FZD6 was strongly expressed mainly in $\mathrm{HCC}$ cells as compared to adjacent nontumorous hepatocytes $(\mathbf{C})$. In the normal liver, no expression was detected either in hepatocytes or normal biliary epithelial cells (D). FZD7 was highly expressed by HCC cells in a well-differentiated HCC (E). In the normal liver, faint labelling was observed in hepatocytes located in the perivenous region of the lobule $(\mathbf{F})$. Size bars $=500 \mu \mathrm{m}$.

Table I Steady state levels of mRNAs for FZD3, FZD6, and FZD7 genes as assessed by quantitative real-time RT-PCR in different human hepatoma cell lines in comparison to normal human primary hepatocytes

\begin{tabular}{lccc}
\hline Hepatocytes & FZD3 & FZD6 & FZD7 \\
\hline Normal primary hepatocytes & $+/-$ & + & $+/-$ \\
Hepatoma HepG2 & ++ & ++++ & $+/-$ \\
Hepatoma Hep3B & + & +++++ & + \\
Hepatoma PLC/PRF/5 & ++ & + & ++ \\
Hepatoma Focus & ++ & ++++ & ++ \\
Hepatoma Huh7 & ++ & + & +++
\end{tabular}

As described in Material and Methods, any gene mRNA steady state level was expressed as arbitrary value (AV). A semi-quantitative scale was proposed for gene expression: $(-): \mathrm{AV}=0 ;(+/-): \mathrm{AV}=0$ to $20 ;(+): \mathrm{AV}=2 \mathrm{I}$ to $50 ;(++): \mathrm{AV}=5 \mathrm{I}$ to $100 ;(+++): A V=|0|$ to $|50 ;(++++): A V=| 5 \mid$ to $200 ;(+++++): A V>200$.

is phosphorylated by casein kinase Ia (CKIa) and glycogen synthase kinase $3 \beta$ (GSK3 $\beta$ ), and polyubiquitinated by hTRCP1 or hTRCP2 for degradation by the proteasome. In the presence of canonical WNT signalling, Dishevelled (DVL) is phosphorylated by CKIa and complexes with FZD. Subsequently, $\beta$-catenin is released from phosphorylation by CKIa and GSK3 $\beta$ for stabilisation, 
Table 2 Accumulation of Wnt/Frizzled-signallling dysregulation events (upregulation of FZD3, FZD6, FZD7, WNT3, WNT4, and WNT5A or repression of sFRPI and sFRP5) between HCC tumours (T) and their matched nontumorous counterparts ( $\mathrm{pT}$ ), and increased activity of different potentially Frizzleddependent intracellular cascades in $\mathrm{T}$ in comparison to $\mathrm{pT}$

\begin{tabular}{|c|c|c|c|c|}
\hline $\begin{array}{l}\text { WNT3/4/5A, FZD } 3 / 6 / 7 \text { and sFRP I/5 } \\
\text { dysregulation events in T vs pT (\# HCC ID) }\end{array}$ & $\begin{array}{l}\beta \text {-catenin mutation } \\
\text { status in } \mathrm{T}\end{array}$ & $\begin{array}{l}\text { Increased activity of } \\
\beta \text {-catenin in } T \text { vs } \mathrm{pT}\end{array}$ & $\begin{array}{l}\text { Increased activity } \\
\text { of PKC in T vs PT }\end{array}$ & $\begin{array}{l}\text { Increased activity } \\
\text { of JNK in T vs pT }\end{array}$ \\
\hline
\end{tabular}

FZD3/6, sFRPI/5 vs sFRP5 (no. 50)

None vs none (no. 51$)$

sFRPI vs WNT4 (no. 52)

WNT3 vs none (no. 54)

FZD3/6/7, WNT3/5A vs SFRP5 (no. 60)

FZD6, sFRP5 vs FZD3 (no. 30)

FZD7, WNT3, sFRPI vs WNT3 (no. 25)

FZD7, WNT4 vs none (no. 36)

FZD3/6/7, WNT4/5A vs none (no. 61)

WNT3/4, sFRPI/5 vs FZD7 (no. 9)

FZD3/7, WNT4 vs none (no. II)

FZD7, WNT5A, sFRP5 vs WNT415A (no. 12)

WNT3/5A, sFRPI/5 vs none (no. 13)

FZD6/7, WNT4/5A vs FZD3/7 (no. 17)

FZD $3 / 7$ vs none (no. 14)

FZD7, sFRP5 vs FZD3 (no. 18)

sFRP5 vs WNT3 (no. 16)

FZD7, WNT4 vs none (no. 20)

None vs none (no. 49)

$\begin{array}{ll}- & + \\ - & - \\ - & - \\ - & - \\ - & + \\ - & - \\ - & - \\ - & + \\ - & - \\ - & - \\ - & + \\ - & - \\ - & + \\ - & + \\ - & - \\ - & + \\ - & - \\ - & + \\ - & -\end{array}$

of PKC in T vs PT

of JNK in T vs PT

The $\beta$-catenin activity was assessed by western blot analysis as a ratio between the level of total $\beta$-catenin and phospho- $\beta$-catenin (Thr4I/Ser45). The cytosolic fraction derived from HCC tissues ( $T$ and $\mathrm{pT}$ ) was used for immunoblotting. The $\beta$-catenin gene status was wild type as revealed by the sequencing of exon-3. The PKC and JNK activities were assessed by western blot analysis as a ratio between the levels of pan-phospho-PKC (Ser660) or phospho-JNK (Thrl83/tyrl 85), and pan-PKC or JNK. (+) Activity of each pathway was considered as higher in T vs pT when the western blot analysis value was increased by at least $25 \%$ in $T$ vs pT. Note the increased of activity of one or more pathways in most of $\mathrm{T}$ vs $\mathrm{PT}$.

cytosolic accumulation and nuclear translocation. Nuclear $\beta$-catenin is complexed with T-cell factor/lymphoid enhancer factor (TCF/LEF) family transcription factors and other proteins (BCL9 and BCL9L Legless family docking proteins, and the PYGO1 and PYGO2 PYGO-family coactivators). The TCF/LEF- $\beta$-cateninLegless-PYGO nuclear complex is the effector of the canonical WNT signalling pathway to activate the transcription of target genes such as FGF20, DKK1, WISP1, MYC, and CCND1. Noncanonical WNT signals are transduced through FZD family receptors and coreceptors, such as ROR2 and RYK. Small G proteins (RHOA, RHOU, RAC, and CDC42) and c-jun NH2terminal kinase are the DVL-dependent effector molecules of the noncanonical pathway, whereas nemo-like kinase (NLK) and nuclear factor of activated $\mathrm{T}$ cells (NFAT) are the $\mathrm{Ca} 2+-$ dependent effector molecules of noncanonical pathway. Small G proteins are implicated in the cytoskeletal reorganisation during invasion and metastasis. NLK phosphorylates TCF/LEF family transcription factors to inhibit the canonical WNT signalling pathway. NFAT transcription factor is implicated in the convergent extension during early embryogenesis as well as in the metastasis during carcinogenesis. Noncanonical WNT signalling pathway, transduced to a variety of DVL- or Ca2 +-dependent cascades, is overlapping with the planar cell polarity signalling pathway (Katoh and Katoh, 2007a). It remains questionable the accuracy of the role of these three FZD potentially dependent pathways in human HCC. Evidence is accumulating to the fact that alterations of the $\mathrm{Wnt} / \beta$-catenin pathway, due or unrelated to $\beta$-catenin gene mutation, is a common event in hepatocarcinogenesis and is associated with the clinical and pathological features of the disease (Hsu et al, 2000; Devereux et al, 2001; Laurent-Puig et al, 2001; Wong et al, 2001; Inagawa et al, 2002). As to the PKC and JNK pathways, their role and prevalence of activation remain poorly known in human hepatocarcinogenesis, although some studies have underlined their potentially oncogenic properties (Delpuech et al, 2002; Guo et al, 2005; Wu et al, 2008). In this study, we did observe an increment in activity of one or more of the three WNT/FZD potentially dependent pathways ( $\beta$-catenin, PKC, and JNK) in a majority of HCCs with the accumulation of dysregulations of WNT/FZD elements and the absence of $\beta$-catenin gene mutation, by comparison to their matched $\mathrm{pT}$. These data suggest that the WNT/FZD-mediating signalling may differentially activate these pathways, although other mechanisms independent of the WNT/FZD signalling may interfere.

Whereas certain WNTs appear to play predominant roles in initiating canonical ( $\beta$-catenin) vs noncanonical (PKC and JNK) pathways, the relative extents to which most WNTs or FZDs participate in one and/or the other pathways has not been well established. Many of FZD receptors, including FZD3 and FZD7, are able to activate the canonical $\beta$-catenin pathway, whereas data are not so obvious for FZD6. The noncanonical PKC and JNK pathways have been clearly shown as potentially activated by some FZD family members as FZD7, whereas it remains ambiguous for FZD3 and FZD6 (He et al, 1998; Winklbauer et al, 2001; Kinoshita et al, 2003; Merle et al, 2004; Katoh, 2007; Katoh and Katoh, 2007b). We have previously reported that the FZD7-mediated signalling was able to control the cancerous phenotype of human HCC cell lines through $\beta$-catenin protein regulation, and that one of the Wnt ligand candidate activators is WNT3 (Merle et al, 2004, 2005; Kim et al, 2008). Nothing is clear about the functional interaction between FZD7 and WNT4 or WNT5A. Others have observed that the WNT3/FZD3 combination could activate the canonical $\beta$-catenin pathway in malignant lymphocytes ( $\mathrm{Lu}$ et al, 2004). Although WNT5A was shown as a natural ligand of FZD3 that triggers the PI3K/Akt signal (Kawasaki et al, 2007), nothing is evident about the WNT4/FZD3 interactions. Finally, there is probably a very complex network of interplays between Wnt-signalling elements and the cellular machinery. Wnt signals are context-dependently transduced to the canonical and noncanonical pathways based on the expression profile of $W N T$, $S F R P$, and FZD genes for instance, and the activity of cytoplasmic WNT signal regulators. Additionally, a higher level of complexity is brought on by the fact that switches of noncanonical signalling molecules may occur during embryogenesis and carcinogenesis (Katoh and Katoh, 2007b).

Importantly, our immunohistochemical approach showed a clear and almost exclusive FZD3/6/7-staining in HCC cells in 

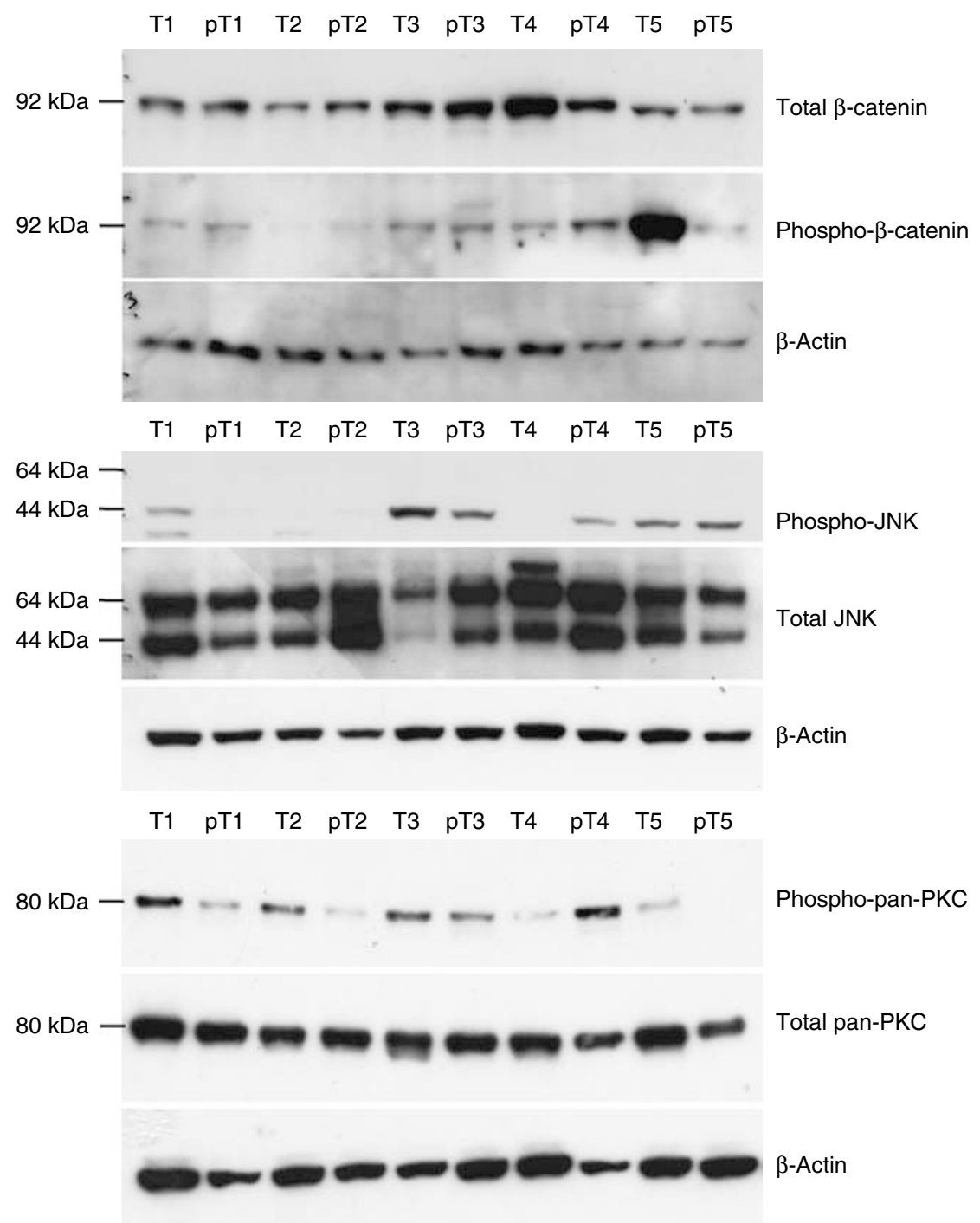

Figure 4 Western blot analysis of FZD-dependent intracellular pathways ( $\beta$-catenin, PKC, and JNK). Activity was assessed in a panel of HCC (T) and their matched nontumorous liver parenchyma (pT). The $\beta$-catenin activity was assessed as the ratio between total- $\beta$-catenin and phsopho- $\beta$-catenin, whereas the PKC and JNK activities were assessed as the ratio between the phospho-PKC or phosphor-JNK and the total PKC or total JNK. All of the blots were standardized for equal protein loading by $\beta$-actin.

tumours and hepatocytes in peritumours, demonstrating that the FZD3/6/7-mediated signalling could be specifically carried out by cancerous cells within HCC tissues, but not by mesenchymal or epithelial biliary cells. In contrast, little is known about the cells secreting the WNT3/4/5 and sFRP1/5 factors within liver tissues, and additional studies would be warranted to identify them as active and/or resting states of various cell types within the liver that is, hepatocytes, biliary epithelial, sinusoidal endothelial, stellate, and Kupffer cells - as previously described in normal mouse livers (Zeng G et al, 2007).

Finally, we have described for the first time that different potential FZD activating events occurring in many human HCC tissues and associated with the activation of different FZD-dependent pathways, which may play a role in hepatocarcinogenesis. This does appear to be an early event that amplifies during liver disease and tumour progression, suggesting a key role in hepatocarcinogenesis. Nonetheless, additional experiments will be required to assess the impact of the different WNT3/4/5A and FZD3/ $6 / 7$ combinations for activation of the FZD-dependent pathways and control of the cancerous phenotype in a specific context-dependent cell state (HCC cells, nontransformed progenitors, and primary cells). This will be a prerequisite before the designation of FZD receptors as exciting targets for therapeutic approaches of HCC.

\section{ACKNOWLEDGEMENTS}

We thank the French National Biological Resources Centre for providing us with the frozen human liver tissues, which were obtained following the approved consent from the French Liver Tumor Network Scientific Committee. The French Liver Tumor Network is funded by the Institut National de la Santé Et de la Recherche Médicale (INSERM) and the Agence Nationale de la Recherche (ANR). This work was supported by French grants from Ligue du Rhône no. 04.18.33), ARC no. 3676), ANRS no. RPF05032CSA), and an interface contract with INSERM for $\mathrm{P}$ Merle as well as grants CA-35711, AA-02066 and P20-RR15578 from the National Institutes of Health (USA).

Supplementary Information accompanies the paper on British Journal of Cancer website (http://www.nature.com/bjc) 


\section{REFERENCES}

Bosch FX, Ribes J, Cleries R, Diaz M (2005) Epidemiology of hepatocellular carcinoma. Clin Liver Dis 9: $191-211$

Breuhahn K, Longerich T, Schirmacher P (2006) Dysregulation of growth factor signaling in human hepatocellular carcinoma. Oncogene 25: $3787-3800$

Delpuech O, Trabut JB, Carnot F, Feuillard J, Brechot C, Kremsdorf D (2002) Identification, using cDNA macroarray analysis, of distinct gene expression profiles associated with pathological and virological features of hepatocellular carcinoma. Oncogene 21: 2926-2937

Devereux TR, Stern MC, Flake GP, Yu MC, Zhang ZQ, London SJ, Taylor JA (2001) CTNNB1 mutations and $\beta$-catenin protein accumulation in human hepatocellular carcinomas associated with high exposure to aflatoxin B1. Mol Carcinog 31: 68-73

Guo L, Guo Y, Xiao S, Shi X (2005) Protein kinase p-JNK is correlated with the activation of AP-1 and its associated Jun family proteins in hepatocellular carcinoma. Life Sci 77: 1869-1878

He TC, Sparks AB, Rago C, Hermeking H, Zawel L, Da Costa LT, Morin PJ, Vogelstein B, Kinzler KW (1998) Identification of c-MYC as a target of the APC pathway. Science 5382: $1438-1441$

Holcombe RF, Marsh JL, Waterman ML, Lin F, Milovanovic T, Truong T (2002) Expression of Wnt ligands and Frizzled receptors in colonic mucosa and in colon carcinoma. Mol Pathol 55: 220-226

Hsu HC, Jeng YM, Mao TL, Chu JS, Lai PL, Peng SY (2000) $\beta$-catenin mutations are associated with a subset of low-stage hepatocellular carcinoma negative for hepatitis $B$ virus and with favorable prognosis. Am J Pathol 157: $763-770$

Inagawa S, Itabashi M, Adachi S, Kawamoto T, Hori M, Shimazaki J, Yoshimi F, Fukao K (2002) Expression and prognostic roles of $\beta$-catenin in hepatocellular carcinoma: correlation with tumor progression and postoperative survival. Clin Cancer Res 8: 450-456

Jones SE, Jomary C (2002) Secreted Frizzled-related proteins: searching for relationships and patterns. BioEssays 24: $811-820$

Katoh M (2007) Networking of WNT, FGF, Notch, BMP, and Hedgehog Signaling Pathways during Carcinogenesis. Stem Cell Rev 3: 30-38

Katoh M, Katoh M (2007a) WNT Signaling pathway and stem cell signaling network. Clin Cancer Res 13: 4042-4045

Katoh M, Katoh M (2007b) Comparative integromics on non-canonical WNT or planar cell polarity signaling molecules: transcriptional mechanism of PTK7 in colorectal cancer and that of SEMA6A in undifferentiated ES cells. Int J Mol Med 20: 405-409

Kawasaki A, Torii K, Yamashita Y, Nishizawa K, Kanekura K, Katada M, Ito M, Nishimoto I, Terashita K, Aiso S, Matsuoka M (2007) Wnt5a promotes adhesion of human dermal fibroblasts by triggering a phosphatidylinositol-3 kinase/Akt signal. Cell Signal 19: 2498-2506

Kim M, Lee HC, Tsedensodnom O, Hartley R, Lim Y-S, Yu E, Merle P, Wands JR (2008) Functional interaction between Wnt3 and Frizzled-7 leads to activation of the $\mathrm{Wnt} / \beta$-catenin signaling pathway in hepatocellular carcinoma cells. J Hepatol 48: 780-791

Kinoshita N, lioka H, Miyakoshi A, Ueno N (2003) PKC delta is essential for dishevelled function in a noncanonical Wnt pathway that regulates Xenopus convergent extension movements. Genes Dev 17: $1663-1676$

Kirikoshi H, Sekihara H, Katoh M (2001) Up-regulation of Frizzled-7 (FZD7) in human gastric cancer. Int J Oncol 19: $111-115$

Laurent-Puig P, Legoix P, Bluteau O, Belghiti J, Franco D, Binot F, Monges G, Thomas G, Bioulac-Sage P, Zucman-Rossi J (2001) Genetic alterations associated with hepatocellular carcinomas define distinct pathways of hepatocarcinogenesis. Gastroenterology 120: 1763-1773

Laurent-Puig P, Zucman-Rossi J (2006) Genetics of hepatocellular tumors. Oncogene 25: $3778-3786$

Lee JS, Thorgeirsson SS (2006) Comparative and integrative functional genomics of HCC. Oncogene 25: $3801-3809$

Llovet JM, Burroughs A, Bruix J (2003) Hepatocellular carcinoma. Lancet 362: $1907-1917$
Lu D, Zhao Y, Tawatao R, Cottam HB, Sen M, Leoni LM, Kipps TJ, Corr M, Carson DA (2004) Activation of the Wnt signaling pathway in chronic lymphocytic leukemia. Proc Natl Acad Sci USA 101: 3118-3123

Maloney JA, Tsygankova O, Szot A, Yang L, Li Q, Williamson JR (1998) Differential translocation of protein kinase $\mathrm{C}$ isozymes by phorbol esters, EGF, and II ANG in rat liver WB cells. Cell Physiol 274: 974-982

Merle P, de la Monte S, Kim M, Herrmann M, Tanaka S, Von Dem Bussche A, Kew MC, Trepo C, Wands JR (2004) Functional consequences of Frizzled-7 receptor overexpression in human hepatocellular carcinoma. Gastroenterology 127: 1110-1122

Merle P, Kim M, Herrmann M, Gupte A, Lefrançois L, Califano S, Trépo C, Tanaka S, Vitvitski L, de la Monte S, Wands JR (2005) Oncogenic role of the Frizzled-7/ $\beta$-catenin pathway in hepatocellular carcinoma. J Hepatol 43: $854-862$

Pasca di Magliano M, Biankin AV, Heiser PW, Cano DA, Gutierrez PJ, Deramaudt T, Segara D, Dawson AC, Kench JG, Henshall SM, Sutherland RL, Dlugosz A, Rustgi AK, Hebrok M (2007) Common activation of canonical Wnt signaling in pancreatic adenocarcinoma. PLoS ONE 2: e1155

Qiang YW, Endo Y, Rubin JS, Rudikoff S (2003) Wnt signaling in B-cell neoplasia. Oncogene 22: 1536-1545

Shih YL, Hsieh CB, Lai HC, Yan MD, Hsieh TY, Chao YC, Lin YW (2007) SFRP1 suppressed hepatoma cells growth through Wnt canonical signaling pathway. Int J Cancer 121: 1028-1035

Shih YL, Shyu RY, Hsieh CB, Lai HC, Liu KY, Chu TY, Lin YW (2006) Promoter methylation of the secreted frizzled-related protein 1 gene SFRP1 is frequent in hepatocellular carcinoma. Cancer 107: $579-590$

Suzuki H, Watkins DN, Jair KW, Schuebel KE, Markowitz SD, Chen WD, Pretlow TP, Yang B, Akiyama Y, Van Engeland M, Toyota M, Tokino T, Hinoda Y, Imai K, Herman JG, Baylin SB (2004) Epigenetic inactivation of SFRP genes allows constitutive WNT signaling in colorectal cancer. Nat Genet 36: 417-422

Tanaka S, Akiyoshi T, Mori M, Wands JR, Sugimachi K (1998) A novel Frizzled gene identified in human esophageal carcinoma mediates APC/ $\beta$-catenin signals. Proc Natl Acad Sci USA 95: 10164-10169

The French METAVIR Cooperative Study Group (1994) Intraobserver and interobserver variations in liver biopsy interpretation in patients with chronic hepatitis C. Hepatology 20(1 Part 1): 15-20

Vincan E, Darcy PK, Smyth MJ, Thompson EW, Thomas RJ, Phillips WA, Ramsay RG (2005) Frizzled-7 receptor ectodomain expression in a colon cancer cell line induces morphological change and attenuates tumor growth. Differentiation 73: $142-153$

Weeraratna AT, Jiang Y, Hostetter G, Rosenblatt K, Duray P, Bittner M, Trent JM (2002) Wnt5a signaling directly affects cell motility and invasion of metastatic melanoma. Cancer Cell 3: 279-288

Winklbauer R, Medina A, Swain RK, Steinbeisser H (2001) Frizzled-7 signaling controls tissue separation during Xenopus gastrulation. Nature 413: $856-860$

Wong CM, Fan ST, Ng IO (2001) $\beta$-catenin mutation and over-expression in hepatocellular carcinoma: clinicopathologic and prognostic significance. Cancer 92: 136-145

Wu TT, Hsieh YH, Hsieh YS, Liu JY (2008) Reduction of PKC-alpha decreases cell proliferation, migration, and invasion of human malignant hepatocellular carcinoma. J Cell Biochem 103: 9-20

Zeng G, Awan F, Otruba W, Muller P, Apte U, Tan X, Gandhi C, Demetris AJ, Monga SP (2007) Wnt'er in liver: expression of Wnt and Frizzled genes in mouse. Hepatology 45: 195-204

Zeng ZY, Zhou YH, Zhang WL, Xiong W, Fan SQ, Li XL, Luo XM, Wu MH, Yang YX, Huang C, Cao L, Tang K, Qian J, Shen SR, Li GY (2007) Gene expression profiling of nasopharyngeal carcinoma reveals the abnormally regulated Wnt signaling pathway. Hum Pathol 38: $120-133$ 